\title{
Affective and cognitive prefrontal cortex projections to the lateral habenula in humans
}

\author{
Karin Vadovičová * \\ Neuroradiology Unit, Department of Diagnostic Imaging, Brescia, Italy
}

\section{Edited by:}

John J. Foxe, Albert Einstein

College of Medicine, USA

Reviewed by:

Ramiro Salas, Baylor College of

Medicine, USA

Martine M. Mirrione, Quinnipiac

University, USA

\section{*Correspondence:}

Karin Vadovičová, Neuroradiology

Unit, Department of Diagnostic

Imaging, Spedali Civili, 25123

Brescia, Italy

e-mail: vadovick@tcd.ie
Anterior insula (Al) and dorsal ACC (dACC) are known to process information about pain, loss, adversities, bad, harmful or suboptimal choices and consequences that threaten survival or well-being. Also pregenual ACC (pgACC) is linked to loss and pain, being activated by sad thoughts and regrets. Lateral habenula ( $\mathrm{LHb})$ is stimulated by predicted and received pain, discomfort, aversive outcome, loss. Its chronic stimulation makes us feel worse/low and gradually stops us choosing and moving for the suboptimal or punished choices, by direct and indirect (via rostromedial tegmental nucleus, RMTg) inhibition of dorsal raphe nucleus (DRN) and VTA/SNc. The response selectivity of LHb neurons suggests their cortical input from affective and cognitive evaluative regions that make expectations about bad, unpleasant or suboptimal outcomes. Based on these facts I predicted direct dACC, pgACC and Al projections to LHb, which form part of an adversity processing circuit that learns to avoid bad outcomes by suppressing dopamine and serotonin signal. To test this connectivity I used Diffusion Tensor Imaging (DTI). I found $\mathrm{dACC}, \mathrm{pgACC}, \mathrm{Al}$ and caudolateral OFC (CIOFC) projections to LHb. I predicted no corticohabenular projections from the reward processing regions: medial OFC (mOFC) and ventral ACC (VACC) because both respond most strongly to good, high valued stimuli and outcomes, inducing dopamine and serotonin release. This lack of LHb projections was confirmed for vACC and likely for mOFC. The surprising findings were the corticohabenular projections from the cognitive prefrontal cortex regions, known for flexible reasoning, planning and combining whatever information are relevant for reaching current goals. I propose that the prefrontohabenular projections provide a teaching signal for value-based choice behavior, to learn to deselect, avoid or inhibit the potentially harmful, low valued or wrong choices, goals, strategies, predictions and ways of doing things, to prevent bad or suboptimal consequences.

Keywords: prefrontal cortex, lateral habenula, inhibitory self-control, adversity processing circuit, value-based choice behavior, dopamine signaling

\section{INTRODUCTION}

I examined the cortical input from the affective and cognitive prefrontal regions to the lateral habenula ( $\mathrm{LHb}$ ) in humans. I predicted that AACC, pregenual ACC (pgACC) and anterior insula (AI) activate the $\mathrm{LHb}$ via direct and indirect projections, forming together an adversity processing circuit. This circuit biases learning, thoughts, feelings and behavior towards gradual inhibition of harmful, punished or suboptimal choices by potentiating the D2 loop of ventral striatum (VS) and by suppressing dopamine and serotonin signal via LHb. Lack of dopamine strengthens the inhibitory avoidance learning and inhibitory self-control and weakens the motivation and drive to move and work for goals and rewards (Vadovičová and Gasparotti, 2013; Figure 1). I suggest that overstimulation of LHb causes discomfort and aversion by down-regulating serotonin signaling (Wang and Aghajanian, 1977), lack of which disinhibits AI, dACC, globus pallidus interna (GPi), LHb (presynaptic LHb inhibition found by Shabel et al.,
2012) and pain pathway output, thus potentiates learned helplessness, depression and anxiety. This is supported by rat studies where learned helplessness was eliminated by habenular lesions, and correlated with increased LHb (and lateral septum) metabolic activity and synaptic potentiation (Amat et al., 1998; Li et al., 2011; Mirrione et al., 2014).

I expected no corticohabenular projections from the reward processing regions, as the good, valuable, rewarding choices, appraised by medial OFC (mOFC), tend to move and motivate us to choose, act and go for them - by stimulating the motivational D1 loop of VS and dopaminergic VTA (Figure 1). Similarly, when we are doing well, reaching good outcomes, safety and gains, it is signaled to brain by the ventral ACC (vACC) activation, that generates fulfilment and satisfaction, increases well-being and lightens up mood by inducing serotonin release in the brain (Figure 1). Based on DRN afferents (Peyron et al., 1998; Vertes, 2004) and serotonin dependent antidepressant effect of vACC stimulation 


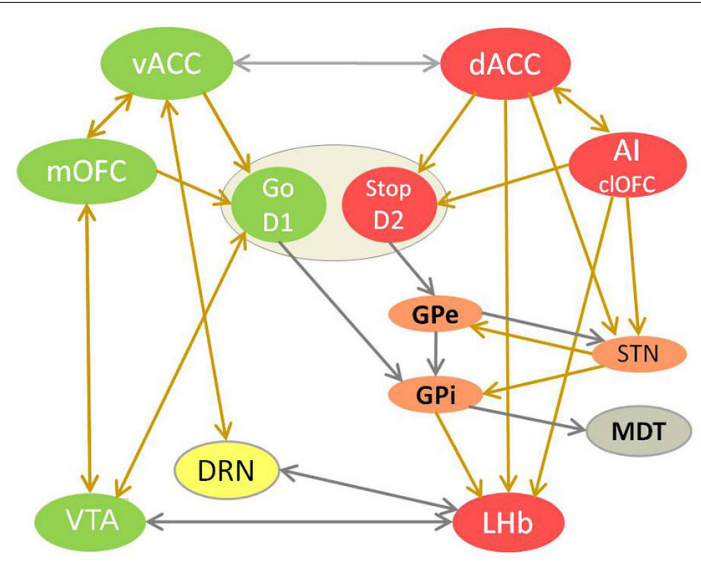

FIGURE 1 | The competition between reward and adversity processing circuit in choice behavior. This model of value-based learning shows how the affective processing causes selection of good/valuable and de-selection of bad/harmful choices, by controling dopamine and serotonin signaling in the brain (Vadovičová and Gasparotti, 2013). The implicit bias/inclination for the "Go for it" vs. "Stop yourself - avoid it" response is learned in the motivational ventral striatum (VS), through potentiation of cortical glutamatergic synapses by dopamine at D1 loop and their depotentiation at D2 loop. Reward and adversity processing circuits are marked green and red. Neurons with either D1 or D2 receptors are spatially intermixed in VS. Ventral striatum neurons with D1 receptors disinhibit dopamine neurons by inhibiting VTA GABA interneurons. The indirect D2 loop (in orange) biases the choice selection toward inhibitory avoidance. The prefrontal projections of MDT enforce the representations of choices and goals in working memory, as part of cortico-striato-thalamo-cortical loop. Dopamine source VTA is marked in green, serotonin source DRN in yellow. The projections with excitatory effects are brown, with inhibitory effect are gray. Dopamine attenuates output of the adversity processing circuit (in red) and potentiates that of reward processing circuit (in green). Serotonin attenuates the Al, dACC, GPi, LHb, SNr, STN plus motivational D1 loop of VS and enforces vACC, SNc (via SNr inhibition), GPe.

in rats (Hamani et al., 2010), I proposed that vACC stimulates DRN directly and via bed nucleus of stria terminalis (BNST). So the LHb is suppressed by dopamine and serotonin, by mOFC to VTA and vACC to DRN projections. In contrary, LHb stimulation and its activation by GPi input (Hong and Hikosaka, 2008) suppresses dopamine signaling in VTA/SNc (Christoph et al., 1986; Jhou et al., 2009; Matsumoto and Hikosaka, 2009; Hong et al., 2011) and serotonin in median raphe nuclei (MRN) and DRN (Wang and Aghajanian, 1977), directly and via the inhibitory rostromedial tegmental nucleus (RMTg). The LHb suppresses also wake/arousal and locomotion promoting histamine release in rats, and opposes its effect on supramamillary nucleus (VanniMercier et al., 1984; Onodera et al., 1994; Kiss et al., 2002). The LHb projects also to noradrenergic locus coeruleus, reticular formation, lateral preoptic area, mediodorsal thalamus (MDT), lateral hypothalamus and superior colliculus (Herkenham and Nauta, 1979). The electrophysiology studies in macaques (Matsumoto and Hikosaka, 2009) showed that LHb neurons respond to punishment cues, unfavorable outcomes and reward omissions. They were most excited by the most negative of the available outcomes, thus firing inversely to the VTA/SNc neurons that are excited by expectation of valuable outcomes. So I claimed that while the reward processing circuit learns about good, valuable choices which increase our well-being and prospects, the adversity processing circuit learns about potentially bad, wrong, harmful or unpleasant choices and outcomes, which decrease well-being and survival chances. Good, interesting things/choices are linked to the approach and motivation to gain them, presumably by preferential mOFC input to D1 loop of VS, while bad things such as pain, harm and loss induce avoidance and aversion, by predominant AI, dACC and pgACC input to its D2 loop. So I extended the basal ganglia learning model (Frank and Hutchison, 2009) by adding the affectively biased cortical input to the VS.

This DTI study is based on the affective processing model that explained how the connectivity of the reward and adversity processing circuits causes their competition, their opposite effects on neuromodulators control, decision making, choice behavior and well-being (Vadovičová and Gasparotti, 2013). This model combined wide evidence from functional, anatomical, dopaminergic, serotonergic and mental dysfunctions studies to specify the interaction of cortical regions with VTA, DRN, LHb, D1 and D2 loop of VS in value-based learning. It stated that dopamine signaling directs and drives us towards valuable, worthy - good, rewarding, interesting, novel, useful, relevant, right and meaningful things, choices and information. This model showed how dopamine guides us to choose, prefer, want, desire, engage with, get interested, inclined, even addicted (in love) to them, and to hope, seek, move, go and work for the valuable, survival and well-being promoting things (food, people, safety, affection, beauty, goals). It hypothesized that serotonin's role is to keep our consumption and wanting within the limits of homeostasis, and to signal when we reached the "comfort zone". Thus the optimal brain serotonin levels promote well-being, fulfilment, satisfaction, feeling well, alright, at ease, non-deprived. They also attenuate drive, motivation, impulsivity, motion and effort, calm down worries, aggression, pain, deprivation and slow us down to rest. The proposed vACC role in signaling gain, well-being, safety, achievement, success is supported by its activation by rewarding outcomes, fear extinction and deactivation by melancholia (Quirk et al., 2003; Pizzagalli et al., 2004; Grabenhorst and Rolls, 2011).

I observed robust dACC and AI co-activation in our NoGo task and in the literature, towards things that are bad or harmful such as pain, loss, risk, suboptimal outcomes, rejection or distress (unpublished M.Sc. thesis). These aversive events decrease well-being and survival chances, so we learn to avoid them by de-selection of choices leading to bad consequences. This deselection is done both consciously - by changing our goals and plans in the medial PFC, and unconsciously - by probabilistic learning of bad or wrong choices by basal ganglia, and by inhibition of dopamine and serotonin release via LHb - consequently affecting all brain regions with dopaminergic or serotonergic receptors. I claim that evaluations and interpretations in the dACC and AI bias the response selection towards inhibitory avoidance by activating the LHb and D2 loop of VS. Dorsal ACC learns, predicts and warns us when we are not doing well, to prevent harm, loss (of resources, loved ones, time) or failure. Its warning signal induces worry, precaution and alarm state, leading to attention, alertness, mobilization (for fight or flight) in risky, 
speed or accuracy demanding situations. This warning signal from dACC urges the PFC to switch away from the inadequate or faulty strategies, to think why things go wrong and find solutions how to change/adjust our world or behavior to stop losing or getting harmed. The AI detects and reacts with aversion to bad, inferior or noxious quality of objects, subjects and social conduct, and also to their moral, conceptual, contextual or task related wrongness. For the pgACC, active in regret, sorrow and sadness (Drevets et al., 1992; George et al., 1995; Brody et al., 2001), I predicted similar LHb projections as for the AACC and AI, leading to passive avoidance.

To test the proposed corticohabenular projections in humans I used the DTI probabilistic tractography. This method does not discriminate the afferent from efferent axonal fibers. But because the tracing studies in animals found only the corticohabenular, no habenulocortical projections, I assumed that the fiber tracts in this study are the LHb afferents. The medial PFC projections to $\mathrm{LHb}$ that regulate dopamine system were shown already in 1982 (Greatrex and Phillipson, 1982) in rats. A tracing study in macaca fuscata found dACC but no vACC/BA 25 projections to LHb (Chiba et al., 2001). Frontohabenular projections were also shown by DTI tractography in humans (Shelton et al., 2012), seemingly originating in BA 10 in their Figure. A retrograde and anterograde tracing study (Kim and Lee, 2012) in rats found corticohabenular projections from the AI, cingulate, prelimbic and infralimbic cortex. The authors found that dense descending projections terminating in the MDT made en passant and terminal projections to the LHb. The PFC is reciprocally connected with MDT, forming cognitive and affective corticothalamo-cortical loops passing via dorsal and ventral striatum (Alexander et al., 1986). The infralimbic cortex in rats is a homolog of vACC in humans, while prelimbic cortex is a homolog of dACC.

\section{MATERIALS AND METHODS}

I used 3 Tesla DTI datasets of 18 healthy participants (24-30 years old) obtained from the NKI Rockland Sample as part of the 1000 Functional Connectomes Project. ${ }^{1}$ The DTI data were acquired with 137 gradient directions, $2 \mathrm{~mm}$ isotropic voxels, 64 slices and FOV $106 \times 90 \mathrm{~mm}$. The T1 weighted anatomical images were acquired with TR/TE/TI $=2500 / 3.5 / 1200 \mathrm{~ms}$, FOV $256 \times 256$ $\mathrm{mm}$, flip angle $8^{\circ}$ and $1 \mathrm{~mm}$ isotropic voxels.

For DTI analysis was used the FSL (FMRIB Software Library ${ }^{2}$ ) version 4.1.9 (Smith et al., 2004; Woolrich et al., 2009; Jenkinson et al., 2012), with a Probtrackx tool (Behrens et al., 2007) for probabilistic tractography. This method generated probabilistic connectivity distributions for the tested axonal projections for each participant. Each DTI dataset has been analyzed independently, using standard FSL procedures. The pre-processing steps included brain extraction, head motion (Jenkinson and Smith, 2001; Jenkinson et al., 2002) and eddy current correction (Behrens et al., 2003). Then Bedpostx tool was applied to calculate diffusion tensor and to model crossing fibers within each voxel of the brain. Default statistical threshold was used

\footnotetext{
${ }^{1}$ http://fcon_1000.projects.nitrc.org/indi/pro/nki.html

${ }^{2} \mathrm{http}: / /$ www.fmrib.ox.ac.uk/fsl/
}

for all analyses. The results were coregistered to the anatomical image and then normalized to the FSL MNI template (MNI152 $2 \mathrm{~mm}$ ). The same procedure was repeated on three additional 1.5 Tesla datasets. The axonal connectivity was analyzed in individual brains instead of in group, to avoid smoothing, which would confound the habenular voxels with adjacent regions.

The seed and target regions for probabilistic tractography were selected manually in the right hemisphere of each brain using the anatomical image (Figure 2). Right hemisphere was chosen as sufficient for my research question, plus some fMRI studies reported its stronger aversive response (Simon-Thomas et al., 2005). The main seed regions of interest were in the AI, dACC and pgACC. The target region was in the LHb, discriminable by its contrast difference due to myelinated fibers. The reward processing $\mathrm{mOFC}$ and $\mathrm{vACC}$ regions were also tested, as I hypothesized their lack of input to LHb. The vACC seed region contained the Brodmann area (BA) 25, which is the posterior part of vACC, plus few voxels adjacent to it but posterior to pgACC. The mOFC seed contained the posterior half of the gyrus rectus, to avoid the adjacent ventral BA 10. The exploratory seed regions sampled the remaining PFC regions: BA 8, 9, 10, 12, 44, 45, 46,47 , to test the input from cognitive prefrontal areas to the $\mathrm{LHb}$. When drawing the LHb seed regions I avoided the voxels adjacent to the ventricle, but included some medial habenula $(\mathrm{MHb})$ voxels, as the $\mathrm{MHb} / \mathrm{LHb}$ border is not discriminable in the anatomical image. This inclusion limits but might not affect my prefrontohabenular connectivity results, as tracing studies found no PFC-MHb connectivity in rats (Herkenham and Nauta, 1977).

\section{RESULTS}

The probabilistic tractography results confirmed the predicted projections from AI, dACC and pgACC to the LHb in all 18 participants (Figures 3-9). Strong interconnectivity was observed between the AI and the adjacent caudolateral OFC (clOFC). Based on their connectivity, they seemed more similar to each other than to other regions. This was found in all 18 studied brains and suggests their common role in inhibitory avoidance.

The lack of corticohabenular projections from the reward processing regions was confirmed in all 18 participants (Figure 10) for vACC and in 15 for mOFC. Questionable, probably disynaptic fiber tracts between $\mathrm{mOFC}, \mathrm{BA} 10$ and $\mathrm{LHb}$ were found in remaining three brains. Because the ventral BA 10 to $\mathrm{LHb}$ tract was adjacent to the reciprocal $\mathrm{BA} 10 / \mathrm{mOFC}$ connections, I could not discriminate these tracts in three brains, for which the results were inconclusive. The indirect, disynaptic tracts in DTI results are caused by misidentification of the fiber tracts crossing same voxel. I found clear disynaptic fiber tract between the mOFC and hypothalamus, and hypothalamus and LHb. My prefrontohabenular connectivity findings were repeated on 1.5 Tesla datasets, using same probabilistic tractography analysis in three additional participants (Figures 11-14).

The prefrontal fiber tracts to the LHb passed mostly via the internal capsule, some via basal ganglia and all via ventromedial part of the anterior thalamus (AM). It is possible that PFC-LHb 


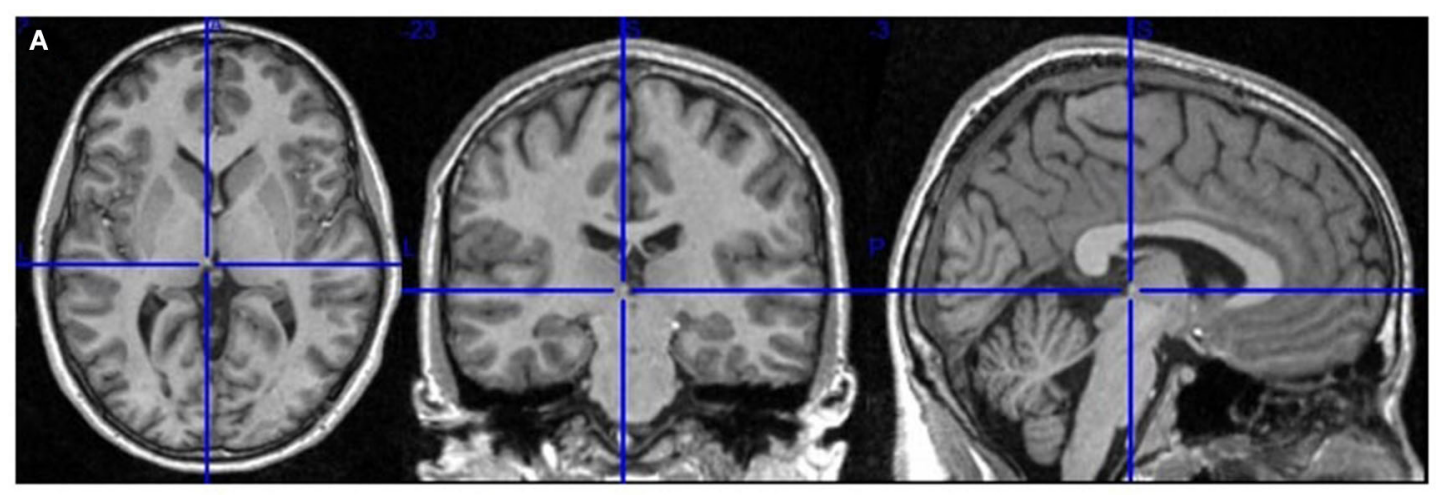

B
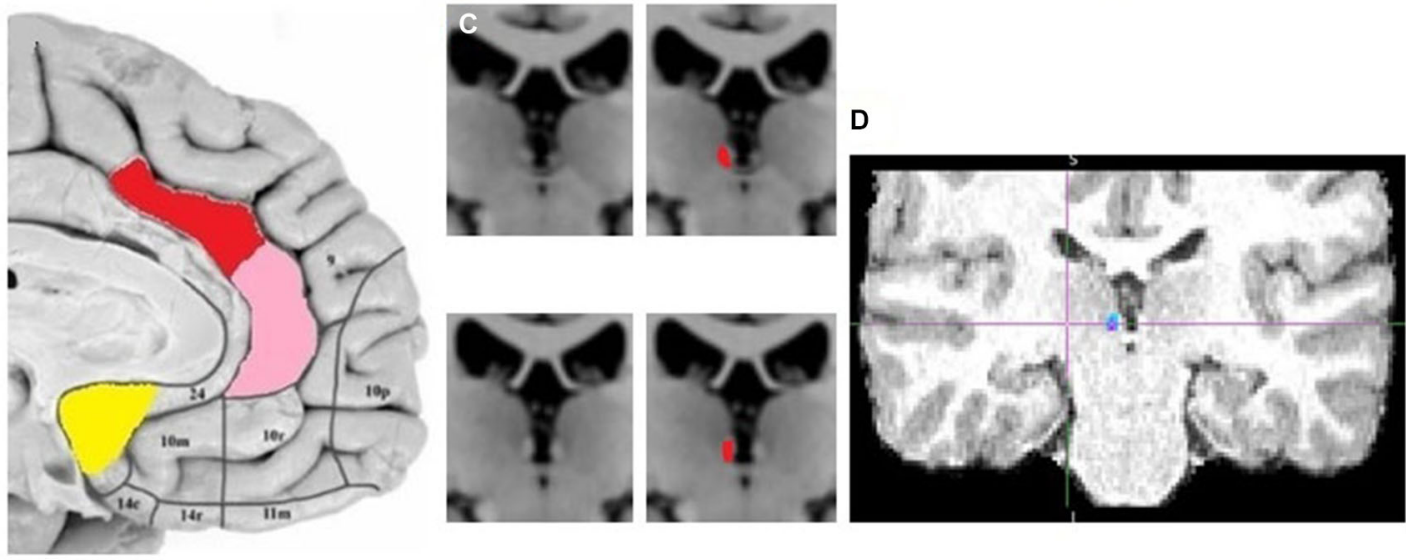

FIGURE 2 | The localization of my ACC seed regions and

habenula. (A) The cross marks the habenula position in the spm anatomical template colin27 (Evans et al., 1993). The coronal section passes through the posterior commissure. (B) The approximate positions of seed regions are shown in sagittal image of human brain from Ongür et al. (2003). In red is dACC seed region (dorsoposterior to genu of corpus callosum), in pink pgACC (anterior to genu), in yellow vACC (ventroposterior to genu). (C) Right and left habenula has lighter contrast than its surrounding. (D) The $\mathrm{LHb}$ region targeted by cortical fiber tract. projections formed collaterals in AM, as they showed second highest fiber density there, after the LHb. The same projections were found after applying the exclusion masks to exclude the MDT and superior colliculus, located above and under the LHb, from the analysis. The dorso-ventral position of the cortical tracts on their way through capsula interna depended on the vertical position of the individual cortical seed regions. So, the ventral $\mathrm{BA} 10$ projections to $\mathrm{LHb}$ crossed striatum between the nucleus accumbens and ventral anterior putamen, forming horizontal fiber tract. But projections from more dorsal regions such as dACC or BA 9 crossed striatum between the lateral caudate nucleus and putamen, forming diagonal tract. The temporal pole (TP) and an additional fiber tract from AI reached the LHb not via capsula interna but by posteriorly localized tracts (Figures 15, 3).

Unexpected findings of this study were the strong projections to $\mathrm{LHb}$ from the cognitive PFC regions: from seeds in the superior, middle and inferior frontal gyrus and from the medial and lateral frontal pole or BA 10. These prefrontohabenular fiber tracts projected from the BA 10, 9, 8, 44, 45, 46, 47, frontal eye fields (FEF) and lateral OFC (Figures 12, 13). I found corticohabenular fiber tracts from all cognitive and affective (AI, clOFC, dACC,
pgACC) PFC regions involved in decision making except the vACC and probably except mOFC.

I showed direct projection between septum and $\mathrm{MHb}$ (Figure 14) passing via BNST and AM and possibly branching there. The $\mathrm{MHb}$ formed the known multisynaptic fiber tract with the hippocampus: hippocampus $\rightarrow$ fornix $\rightarrow$ septum $\rightarrow \mathrm{Hb}$ $\rightarrow$ pineal gland. I found also hypothalamo-LHb fiber tracts in humans, without examining exact nuclei. Similar connectivity in rats includes $\mathrm{LHb}$ afferents from the lateral hypothalamic and preoptic nucleus, plus efferents to the lateral preoptic and supramamillary nucleus, ventrolateral septum, lateral and dorsomedial hypothalamus (Herkenham and Nauta, 1977, 1979).

The following figures show a sample of found fiber tracts. These DTI images are from different individual brains. The left hemisphere in the image represents the right hemisphere of brain. The LHb slice is not always shown, as I prioritized to show fiber tract passage.

\section{DISCUSSION}

Results of DTI tractography support the functional connectivity of proposed adversity processing circuit, formed by dACC, 

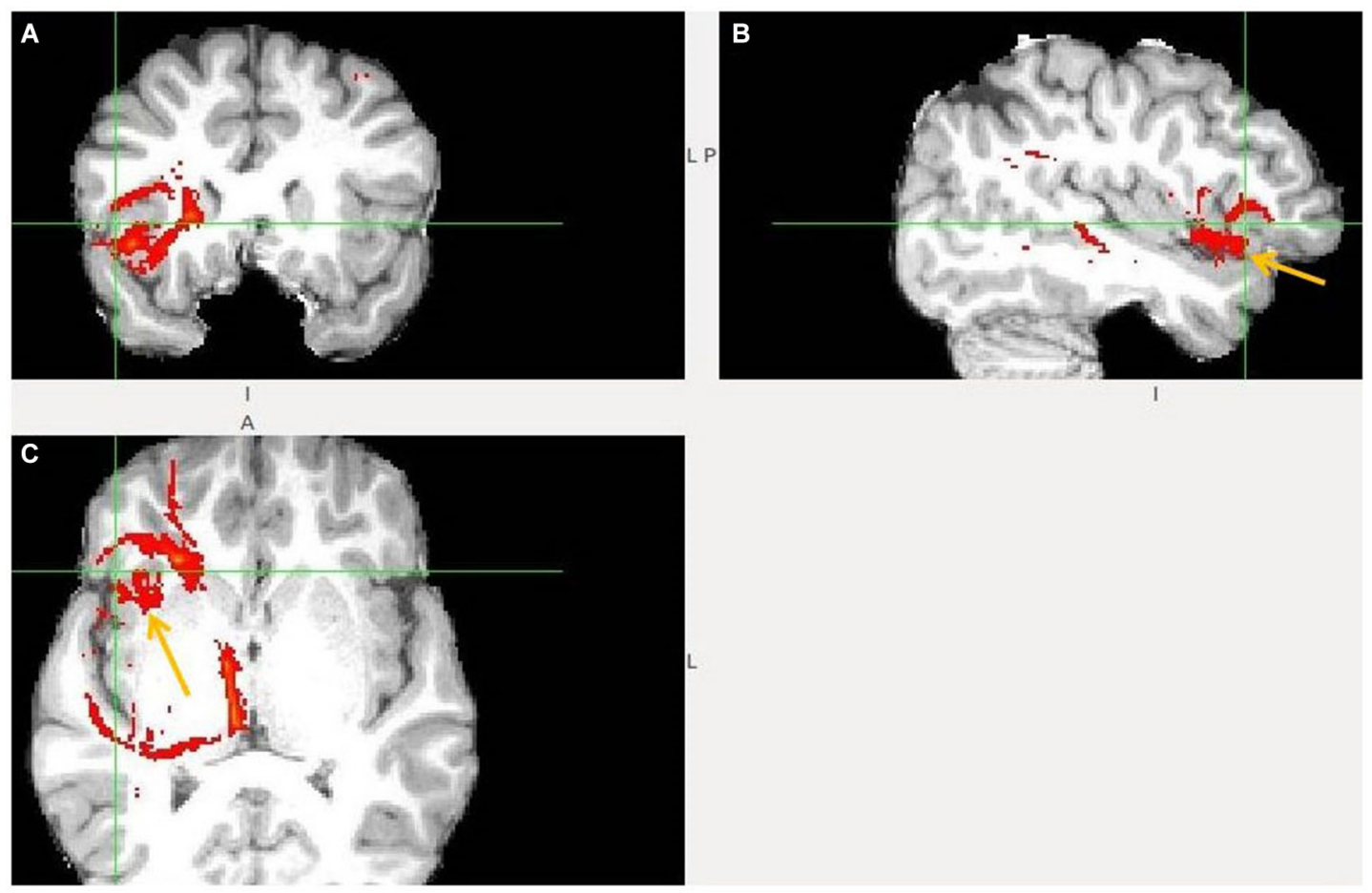

FIGURE 3 | Anterior insula to LHb fiber tract. (A,B) The Al tract is branching to clOFC that is anterior to ventral Al. The arrows point to Al. (C) Visible is branching from Al to temporal pole (TP) and TP to LHb.
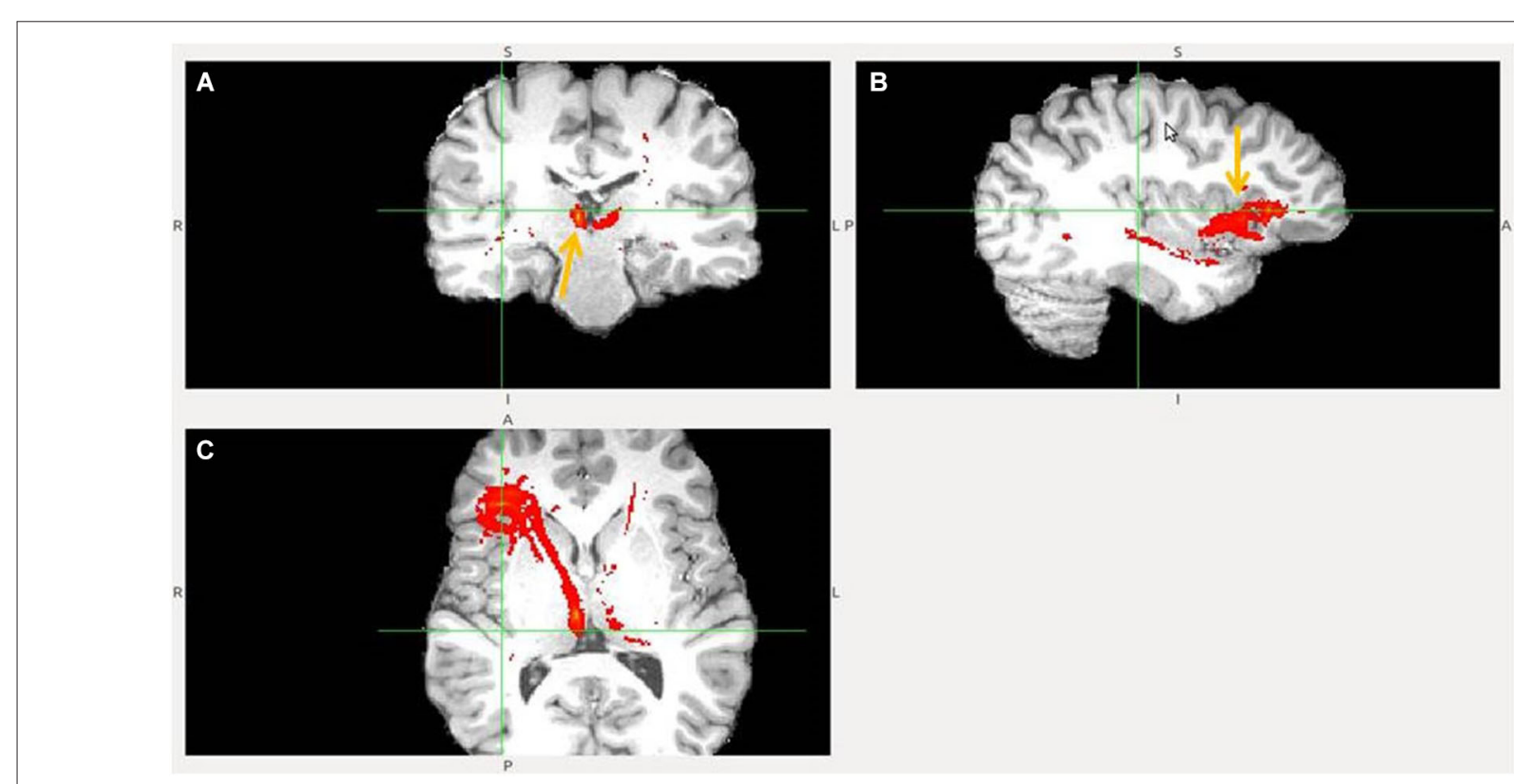

FIGURE 4 | Anterior insula to LHb fiber tract, different slices. (A) Tract termination in right and left habenula (arrow). (B) Anterior insula (arrow) is connected with clOFC and projects to TP. (C) Al/clOFC to LHb.

pgACC, AI and adjacent clOFC input to the LHb. This circuit detects, learns about and predicts potential adversities and forwards the information about bad, harmful or suboptimal choices and consequences to the LHb, to suppress dopamine and serotonin release in the VTA and DRN (Vadovičová and Gasparotti, 2013). The causal role of this corticohabenular circuit in aversive 

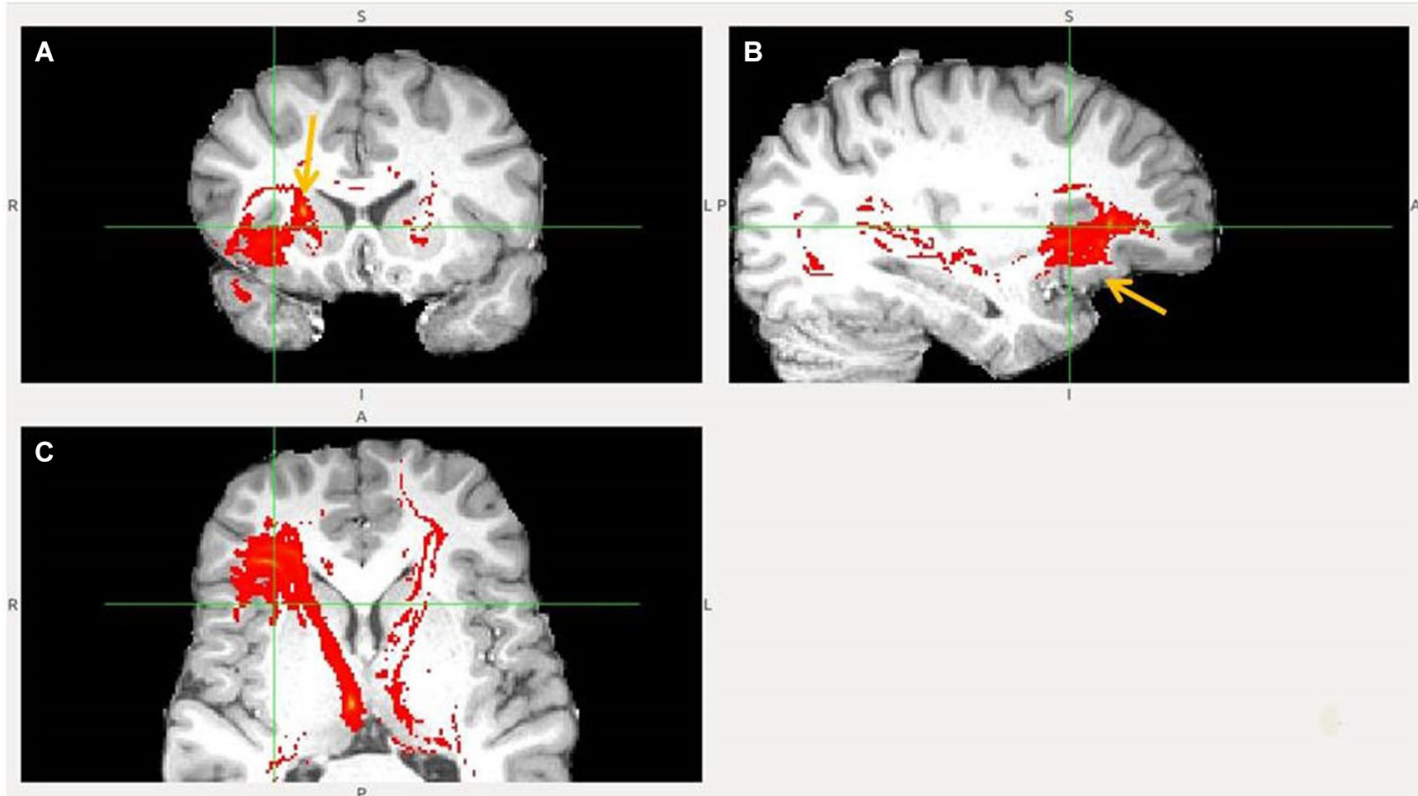

FIGURE 5 | Anterior insula to LHb fiber tract, anoher slices. (A) Anterior insula and clOFC projections to LHb via capsula interna. (B) Anterior insula tract decends to clOFC. (C) Al/clOFC tract targets LHb, shown at its dorsal part.
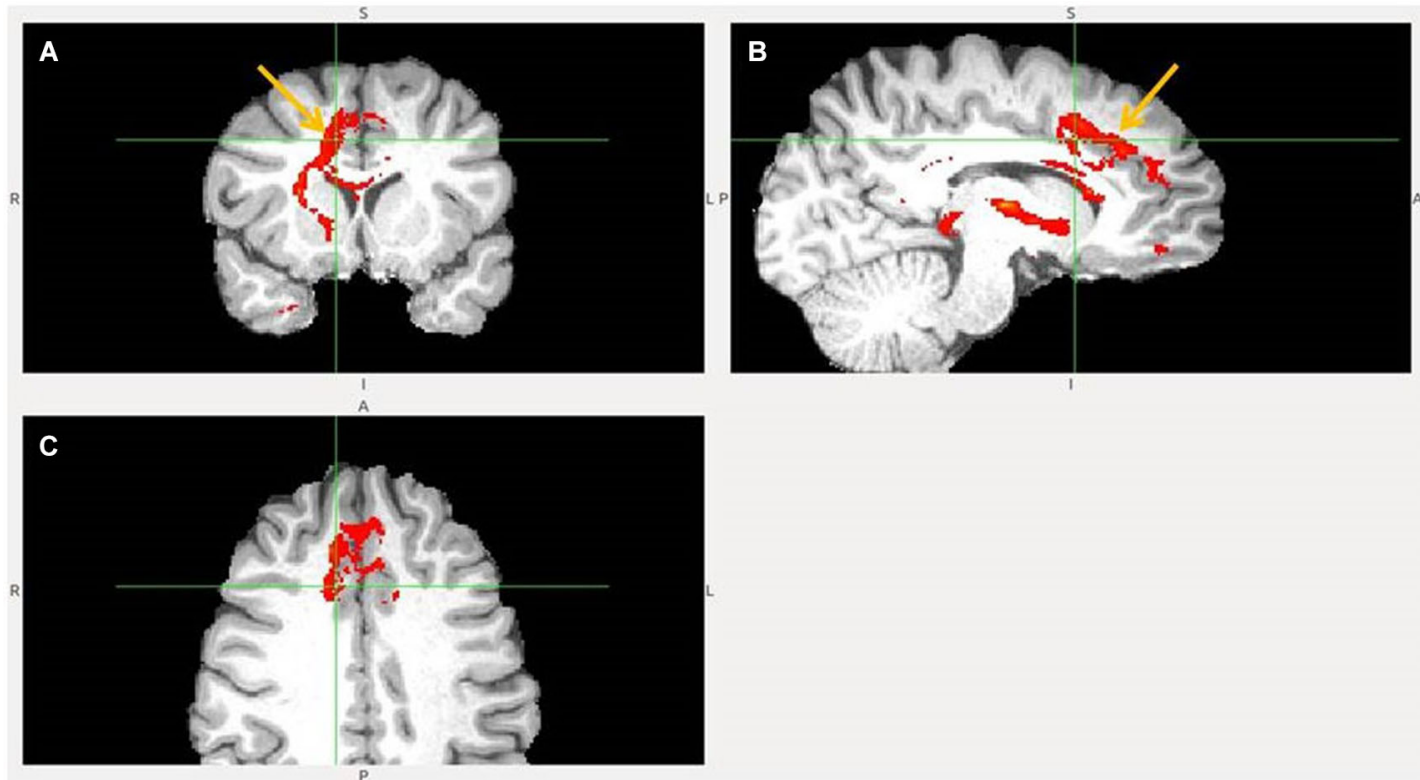

FIGURE 6 | Dorsal ACC to LHb fiber tract. (A) Tract passes via capsula interna. (A,B,C) The images show dACC, pointed to by arrow.

processing is supported by findings from functional, behavioral, pharmacological and mental disorders studies. An example is the co-activation of dACC, AI and LHb during negative feedback (Ullsperger and von Cramon, 2003) in humans. I observed strong interconnectivity between the AI and adjacent clOFC. Based on their shared connectivity and common co-activation in functional tasks in literature, I suggest that the $\mathrm{AI}$ and clOFC form a functional processing module, because connectivity determines function and computational role. Possibly they process similar kind of information about bad - harmful, aversive, inferior, corrupted or 

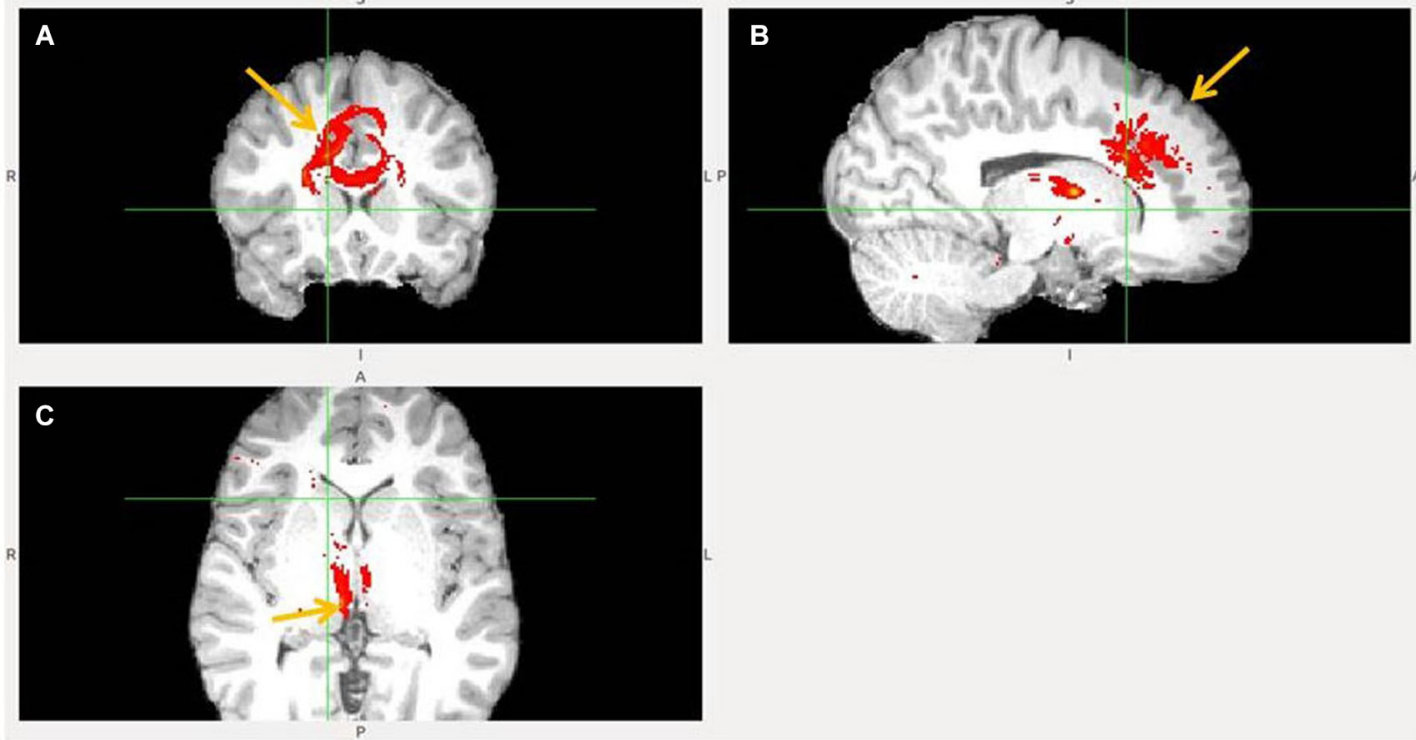

FIGURE 7 | Dorsal anterior cingulate cortex (dACC) to LHb fiber tract, different slices. Arrows indicate dACC (A,B) and LHb (C)
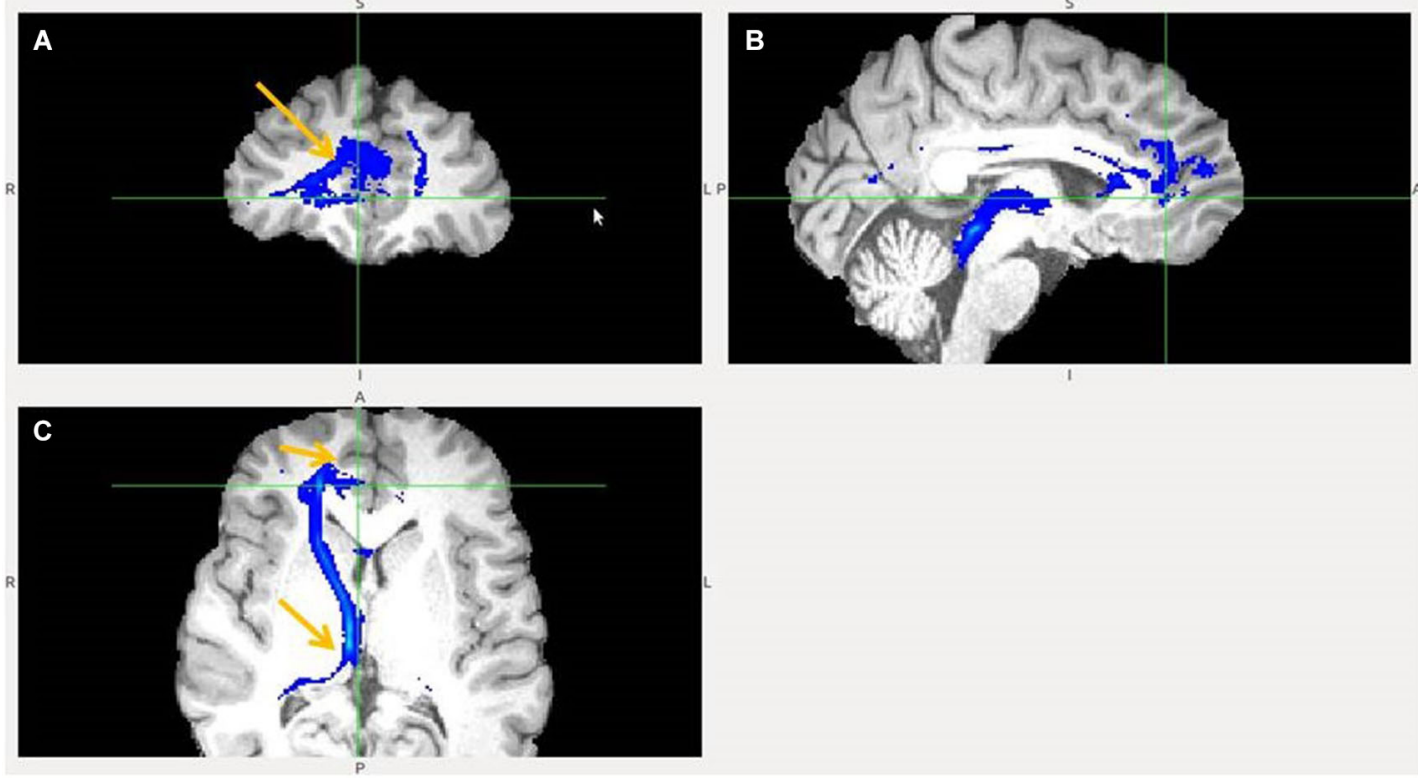

FIGURE 8 | Pregenual anterior cingulate cortex (pgACC) fiber tract to LHb. (A) pgACC pointed by arrow. (B) pgACC is located in front of genu of corpus callosum. This fiber tract passes via

suboptimal qualities/attributes of objects, subjects or conducts. So the $\mathrm{AI} / \mathrm{clOFC}$ probably selectively learns about things of negative or low value - aversive, unpleasant, disliked or safer to avoid. The $\mathrm{AI} / \mathrm{clOFC}$ responds also to the contextual (regarding the current task or situation), conceptual (false, strange, misfit) and moral wrongness of things or conduct.

In accordance with the affective circuit competition model (Vadovičová and Gasparotti, 2013), in which the mOFC and medial anterior thalamus (AM) to $\mathrm{LHb}$, then branches to superior colliculus, PAG, DRN, pons. (C) Arrows indicate ventral part of pgACC and part of tract just above the $\mathrm{LHb}$.
vACC suppress the LHb via VTA and DRN stimulation, I found no direct vACC and likely no direct mOFC projections to LHb. The mOFC fiber tracts were either passing to LHb via hypothalamus, which was clearly not a direct projection, or via the ventral BA 10. The lateral hypothalamus is reciprocally connected with LHb (Herkenham and Nauta, 1977) and the mOFC with hypothalamus and BA 10. The anterior half of gyrus rectus, anteriorly adjacent to mOFC region, belongs to the ventral BA 10. The 

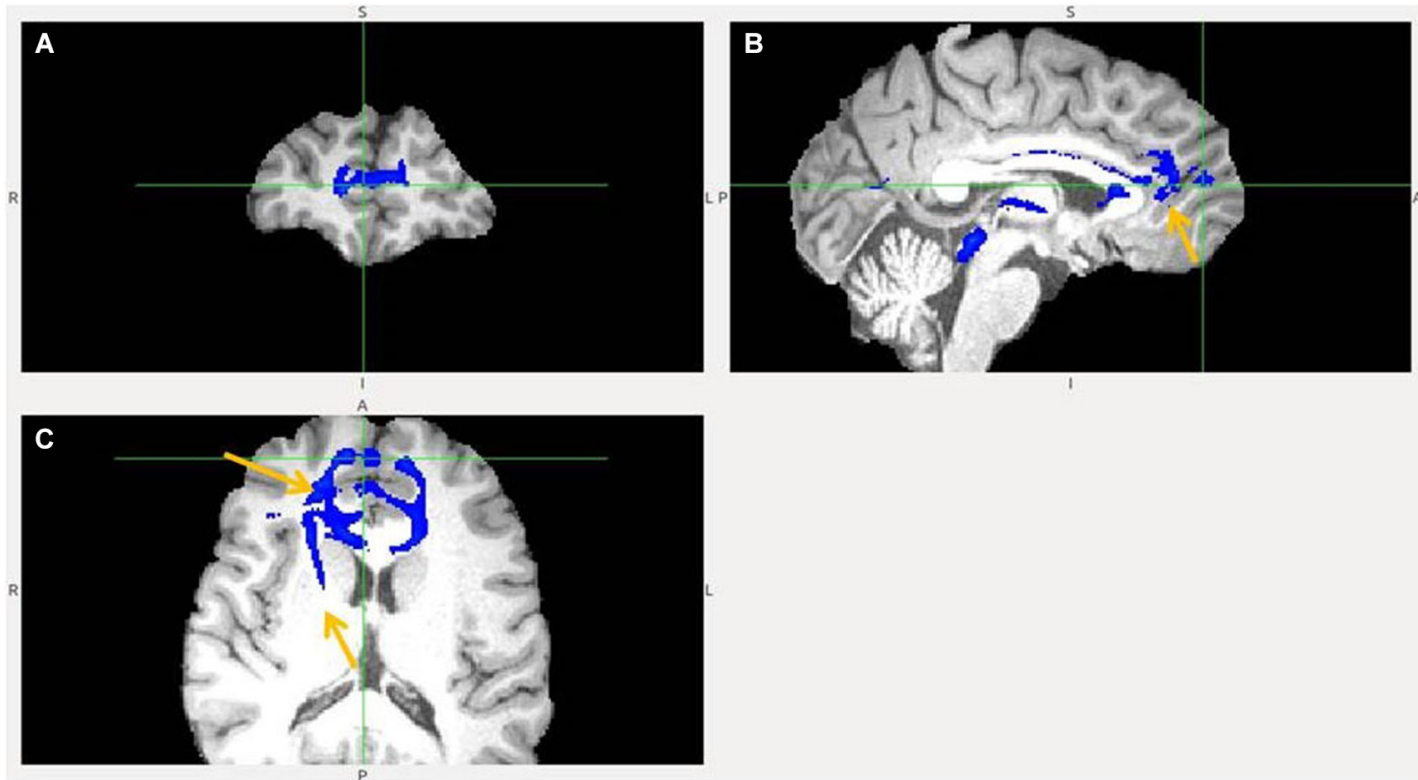

FIGURE 9 | Pregenual anterior cingulate cortex (pgACC) fiber tract to LHb, another slices. (A) Frontal pole cluster where pgACC tract is branching. (B) Arrow points to pgACC part of the tract. Visible is thalamic passage. (C) Arrows show pgACC and capsula interna part of tract.
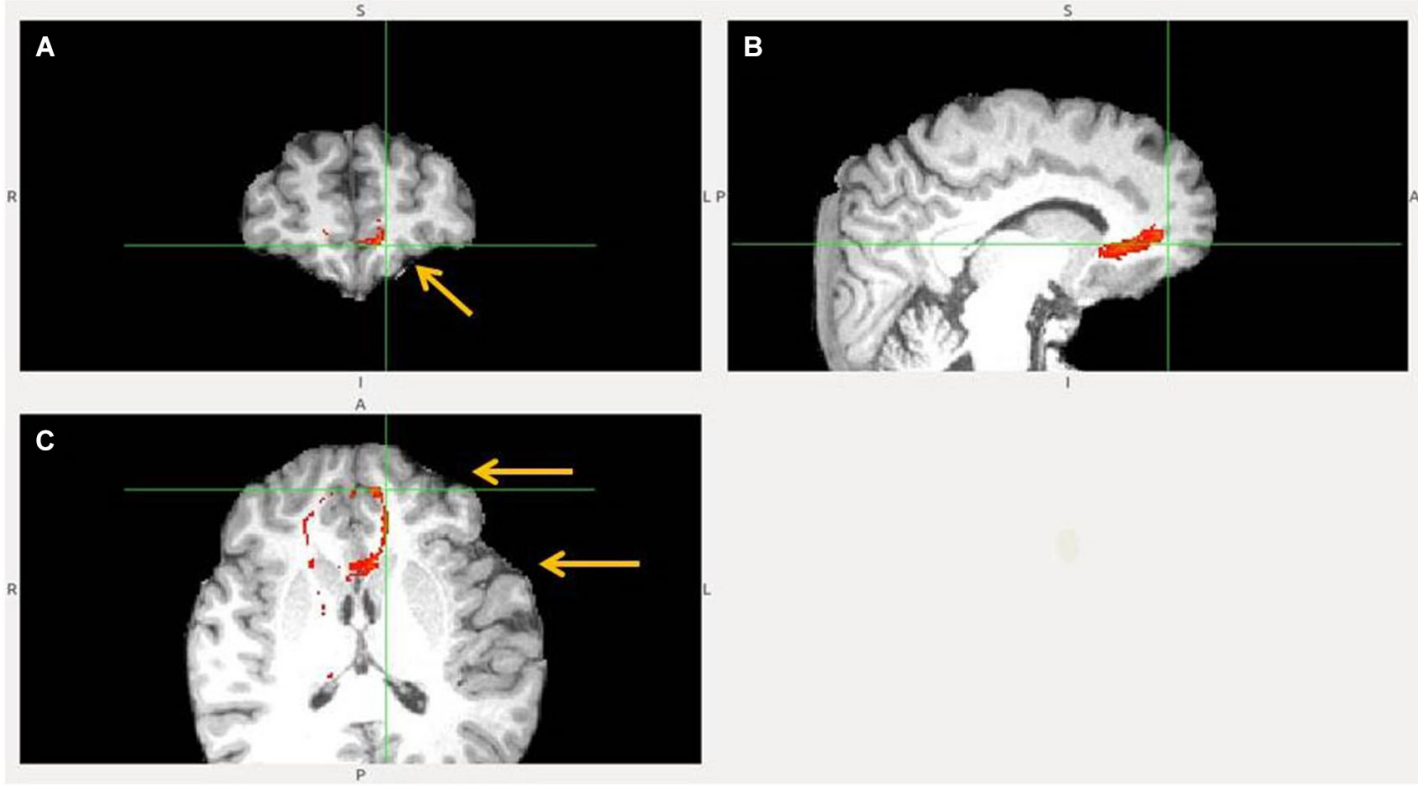

FIGURE 10 | No vACC to LHb fiber tract. This tract (B,C) reached $\mathrm{LHb}$ only indirectly by its projections to BA 10 (A). BA 10 has own robust $\mathrm{LHb}$ projections. Ventral $\mathrm{ACC}$ is indicated by lower

arrow, BA 10 by upper one (C). Ventral ACC tract branched also to hypothalamus, which has reciprocal connection with $\mathrm{LHb}$ (not shown). ventral BA 10 has strong projections to the LHb, so the questionable mOFC to LHb fiber tracts found in 3 of 18 participants were possibly formed by the reciprocal mOFC/BA 10 projections, adjacent to the separate BA 10 to LHb projections. This study supports the opposite effects of the adversity vs. reward processing circuit on the activation vs. inhibition of LHb. Evidence for the reward value coding in mOFC comes from many studies where mOFC responded to rewarding or pleasant stimuli and wins while lateral OFC responded to aversive options, punishment or loss (O’Doherty et al., 2001). 

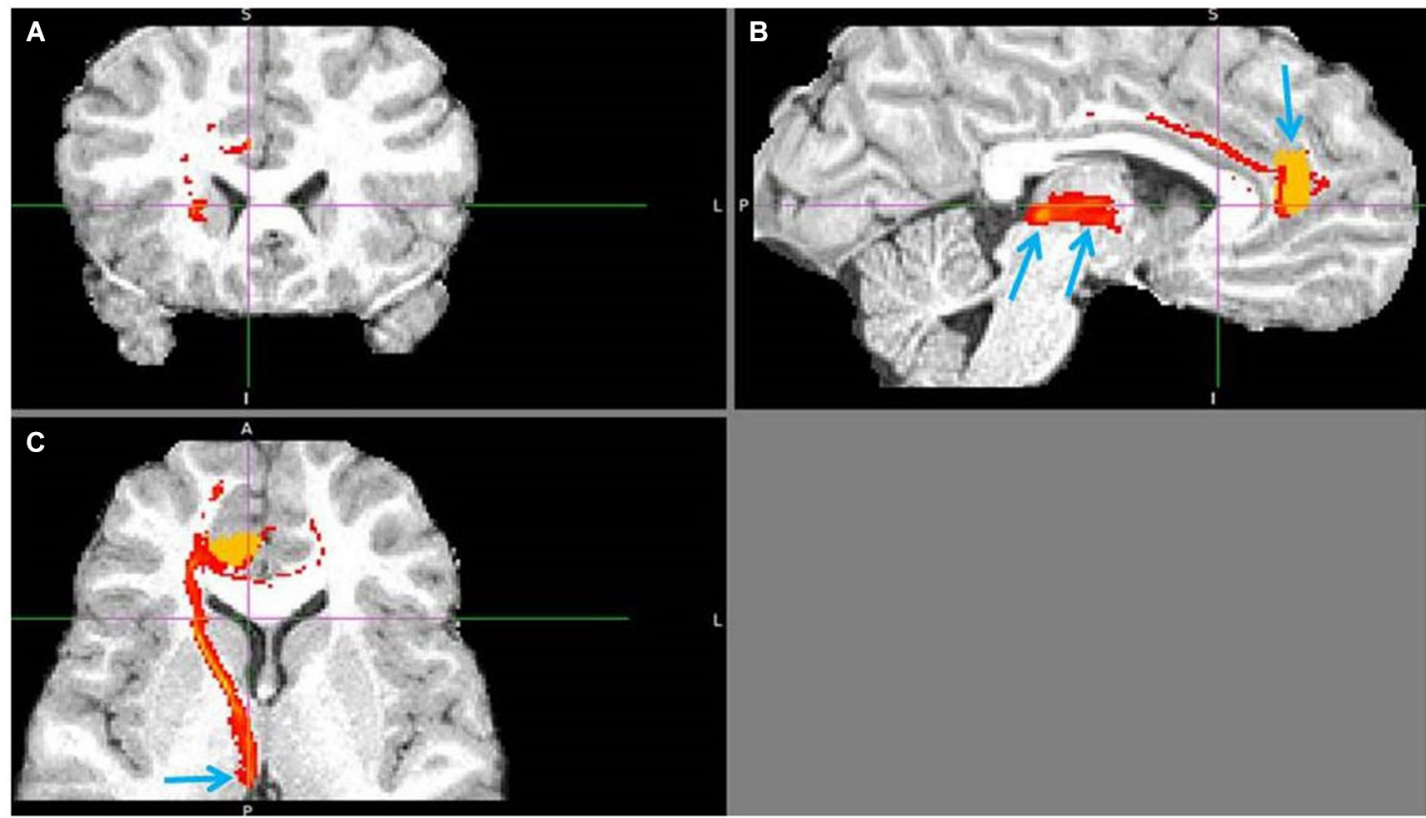

FIGURE 11 | Pregenual anterior cingulate cortex (pgACC) to LHb tract. The fiber tract is in red, pgACC seed region in orange. (A) Passage via capsula interna. (B) Upper arrow points to pgACC seed, bottom left to LHb and bottom right to tract passing via AM. Visible is also the pgACC projection to dACC, which also projects to LHb. (C) Arrow shows where the tract descends into LHb, just 1 slice above it.

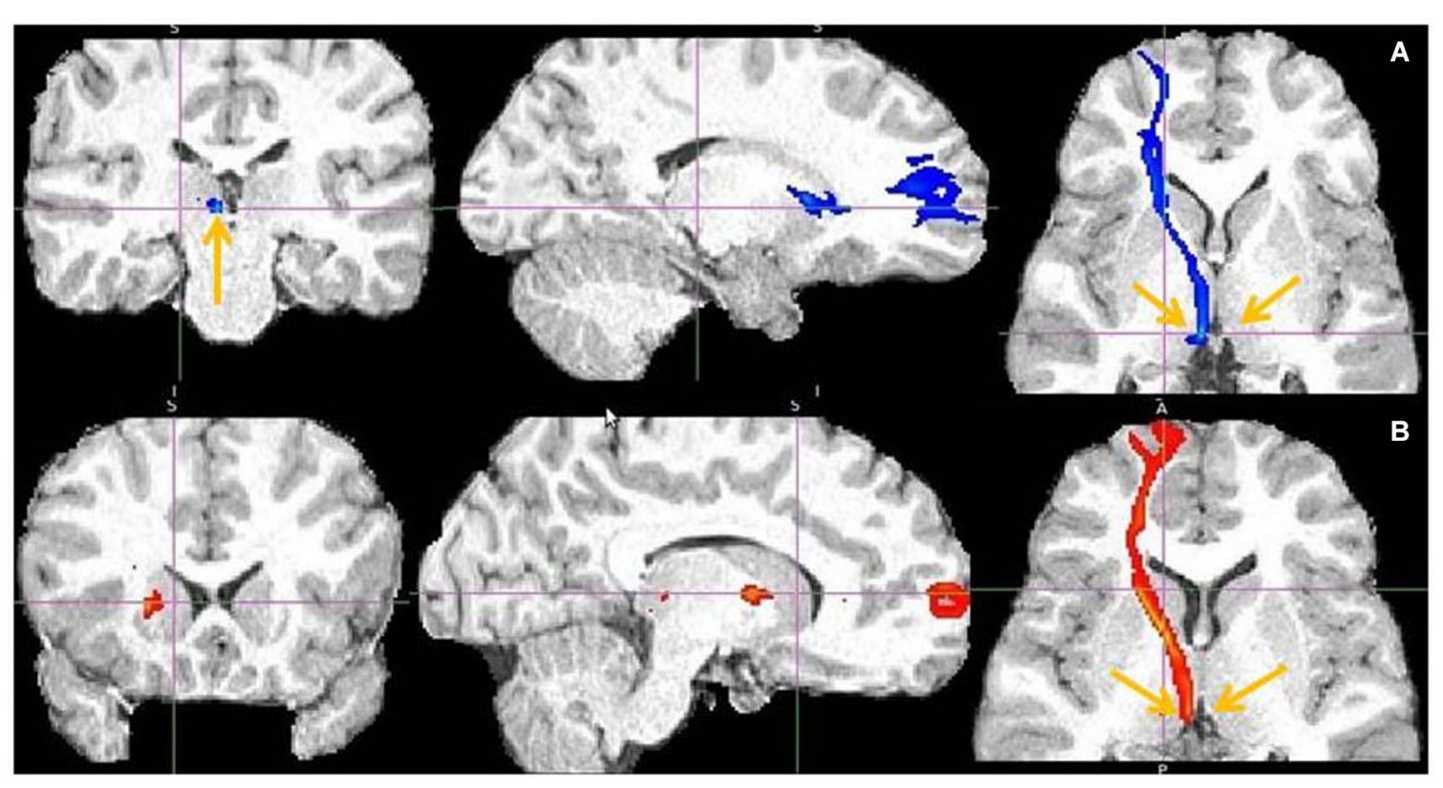

FIGURE 12 | Frontal pole to LHb projection. Arrows point to LHb (A) and to tract just above it (B).

Temporal pole projections to LHb found in this study are likely from the neuronal populations linked to the negative meaning of the represented stimuli, attributes and concepts. Temporal poles process conceptual and semantic information about meanings, properties and identities of things and persons. They are interconnected with BA 10, dACC, vACC, $\mathrm{mOFC,} \mathrm{AI,} \mathrm{thus} \mathrm{receive}$ information about both bad and good values and outcomes linked to objects, subjects or concepts.

I found a multisynaptic fiber tract passing from hippocampus via fornix to septum and from septum to $\mathrm{MHb}$. Supracommissural septum is the main MHb afferent (Herkenham and Nauta, 1977). Septum was connected also with vACC and 


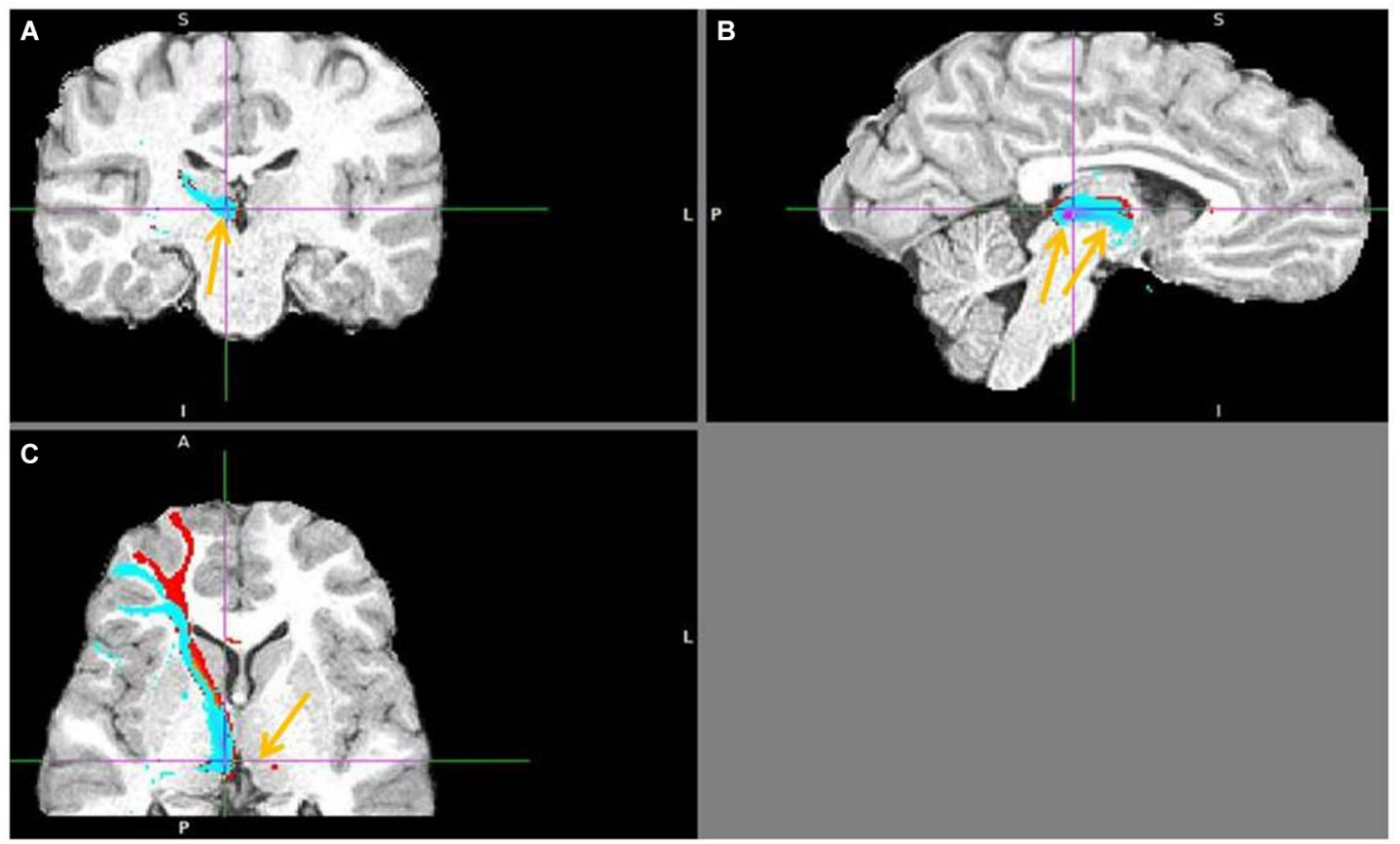

FIGURE 13 | Lateral BA 10 (red) and inferior frontal gyrus IFG (blue) fiber tracts to LHb. (A) Arrow indicates LHb. (B) The highest density of connected fibers is in LHb (purple region). Both seed regions have overlapping tracts via thalamus (right arrow shows AM). (C) Arrow points above LHb.

hypothalamic nuclei in my study. This septo-habenular fiber tract passed via AM and BNST. So septum might also be linked with BNST and BNST with habenula, by afferents, efferents or both. Hippocampus has known efferents from subiculum via fornix to septum, anterior thalamus and mamillary body (MB), and MB also projects to AM (Aggleton et al., 2005). Supramamillary nucleus, which projects to medial septum and via fornix to hippocampus, receives afferents from LHb, medial and lateral septum, preoptic areas, interpeduncular nucleus (IPn), MRN, DRN and laterodorsal tegmentum (LDT), (Kiss et al., 2002). Supramamillary nucleus is known to stimulate theta rhythm during exploration in rats (Vertes and Kocsis, 1997) that gets disrupted by MRN serotonin. The MHb receives input from glutamatergic, cholinergic or substance $\mathrm{P}$ neurons of the triangular septal and septofimbral nucleus, from GABAergic medial septum and diagonal band nucleus, dopaminergic VTA, serotonergic raphe and noradrenergic locus coeruleus neurons (Herkenham and Nauta, 1977; Gottesfeld, 1983; Qin and Luo, 2009). Medial habenula projects to the LHb (Kim and Chang, 2005), pineal body (Rønnekleiv and Møller, 1979), IPn and via IPn to MRN and LDT in rats (Herkenham and Nauta, 1979; Groenewegen et al., 1986). The MHb pathway regulates sleep cycle (Hikosaka, 2010). Based on its anatomical connectivity and interactions with the neuromodulators I propose that $\mathrm{MHb}$ stimulates the non-REM sleep via IPn and MRN, suppressing the rapid eye movement (REM), theta and alertness driving regions. This is supported by dense mu opioid receptors and circadian rhythmicity of MHb neurons (McCormick and Prince, 1987; Quick et al., 1999; Guilding and Piggins, 2007), by the markedly increased $\mathrm{MHb}$ or LHb activity during anesthesia (Herkenham, 1981; Abulafia et al., 2009; van Nieuwenhuijzen et al., 2009), by the fact that the $\mathrm{MHb}$ neurons produce melatonin ( $\mathrm{Yu}$ et al., 2002) and sleep promoting interleukin IL-18 (Sugama et al., 2002) and control via IPn the median raphe serotonin (Wang and Aghajanian, 1977; Agetsuma et al., 2010). Further evidence comes from high firing rates of MRN cells in non-exploratory waking states (when not recording new information in hippocampus) and slow-wave sleep (SWS), plus their low firing rates during the exploration and in REM sleep (theta states), (Jacobs and Azmitia, 1992; Marrosu et al., 1996). Serotonergic DRN neurons, noradrenaline and histamine neurons fire most during wakefulness, less during SWS and are suppressed in REM sleep (Hobson et al., 1975; McCarley and Hobson, 1975; Pace-Schott and Hobson, 2002), while cholinergic activity in LDT and pedunculopontine tegmental nuclei (PPT) is high at wake and REM sleep.

The unexpected findings of this study were the robust projections to $\mathrm{LHb}$ from the cognitive PFC regions, known for flexible coding, combining and holding in working memory any kind of relevant information useful for current goals and tasks (Rainer et al., 1998; Asaad et al., 2000). The strongest prefrontohabenular fiber tracts were from the frontal pole, also known as BA 10. Brodmann area 10 is interconnected with all $\mathrm{PFC}$ regions plus with the associative cortex in the temporal poles and superior temporal gyrus - so well informed and suited for flexible learning, reasoning, planning and goal-directed control of behavior. The medial BA 10 is interconnected with the hippocampus and linked to temporal context, introspection, 


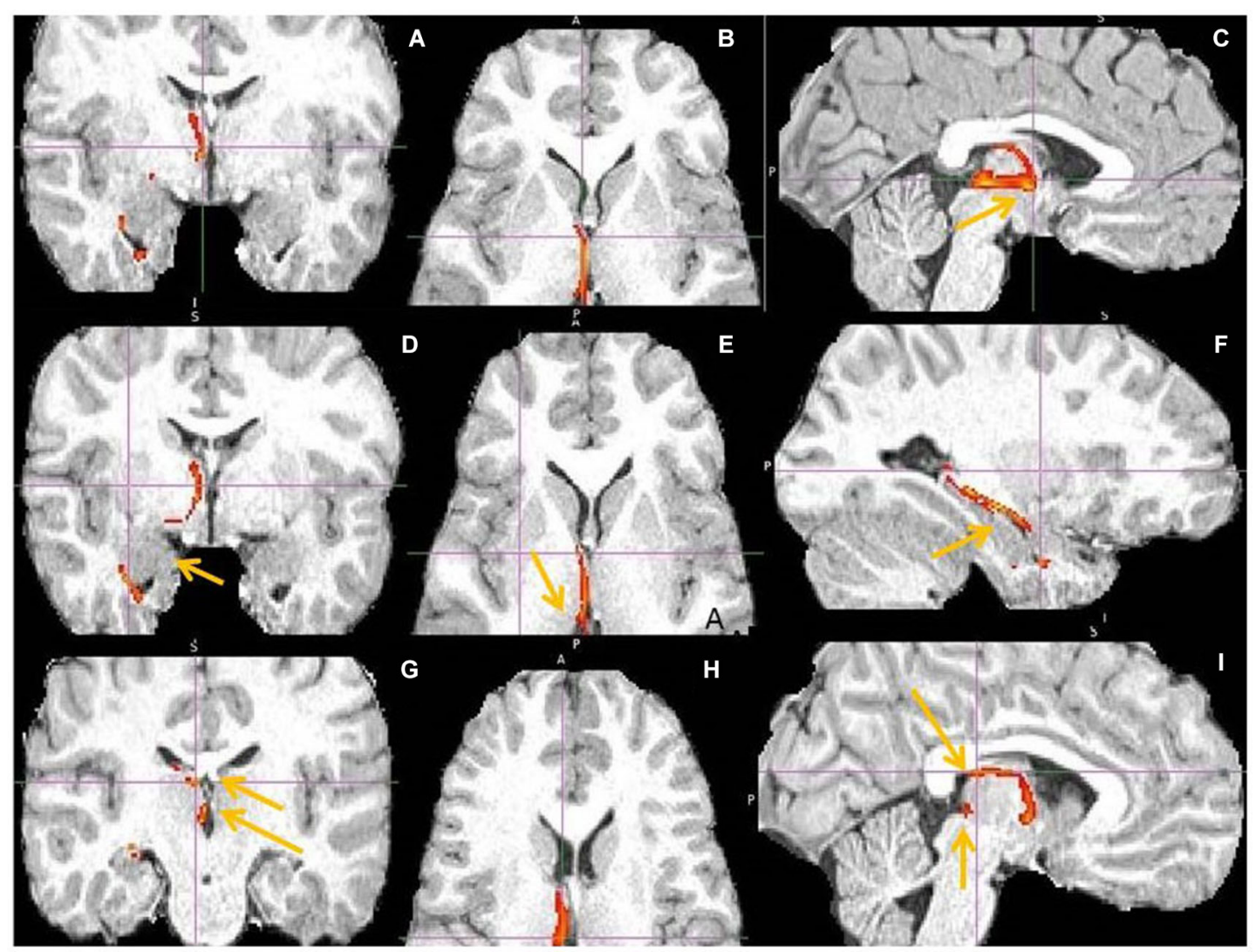

FIGURE 14 | hippocampus to medial habenula (MHb) fiber tract. This fiber tract includes: hippocampus $\rightarrow$ fornix $\rightarrow$ septum $\rightarrow \mathrm{MHb} \rightarrow$ pineal gland. The tract between septum and habenula passes via AM and via bed nucleus of stria terminalis (BNST), where it might send axon collaterals. The AM has the second highest fiber density in this tract. The BNST might connect both septum and habenula via afferents, efferents or both. Fornix has known projections to septum, anterior thalamus and mamillary body $(\mathrm{MB})$ and septum to $\mathrm{MHb}$. (A,D) Tract passage via septum and AM. Arrow marks amygdala, linked by tract with hippocampus. (B,E,H) Tract passage via AM to $\mathrm{MHb}$, and $\mathrm{MHb}$ to pineal gland. Arrow marks $\mathrm{MHb}$. (C,I) Tract passage via fornix to septum, and septum via AM to $\mathrm{MHb}$. Arrow in (C) points to AM, in (I) to fornix and MHb. (F) Tract passage via hippocampus, towards fornix posteriorly and amygdala anteriorly. (G) The arrows point to septum (upper) and $\mathrm{MHb}$ (lower). intentions, goals and planning - so to hierarchical temporal organization of our thoughts. The lateral BA 10 or rostrolateral PFC, stimulated by informational novelty and problem solving, is most extended in humans. It probably induces dopamine release in the medial SNc that stimulates novelty seeking via motivational D1 loop of VS and goal-pursuit via D1 loop of medial head of caudate. I propose that lateral BA 10 role is to seek and find out what is going on - the links between causes and consequences, contingencies, patterns, rules - to make cognitive predictions about our world, test them, then apply the right guesses/hypotheses and ways of doing things to reach our goals. The dorsal PFC is linked to spatial context, spatial organization, planning and control of behavior, while the ventrolateral PFC is guiding the thoughts and behavior by using the meanings, ideas and interrelations between things, actions and events.

The robust input from the cognitive PFC to the LHb possibly enables the inhibitory self-control and context/goal dependent de-selection of wrong, irrelevant or inappropriate information, ideas, decisions, plans, strategies or ways of doing things, depending on the current task or goal. All prefrontal regions generate predictions about the world. The affective regions predict the reward value of choices and consequences, to bias decision making and selection of goals/intentions in the medial BA 10. These goals are then used by all PFC regions to plan and guide the execution of behavior to reach goals and avoid harm or loss. The cognitive regions predict the informational value and cognitive significance of things and events - what is the optimal choice or solution, right or wrong, useful, interesting, what has a predictive value.

Based on found connectivity and wide literature data I propose that affective prefrontohabenular input inhibits VTA, leading to potentiation of the D2 loop of VS, causing inhibition, passive avoidance and de-selection of harmful or suboptimal choices. Similarly, the cognitive prefrontohabenular input inhibits SNc, serving as teaching signal that potentiates the D2 loop of caudate head, to gather evidence on what went wrong, failed or was incorrect, to bias decisions via cortico-striato-thalamo-cortical loop. So the right, correct, valid predictions, ideas, models, strategies and ways of doing things (to reach goals), the "know how", IF-THEN 

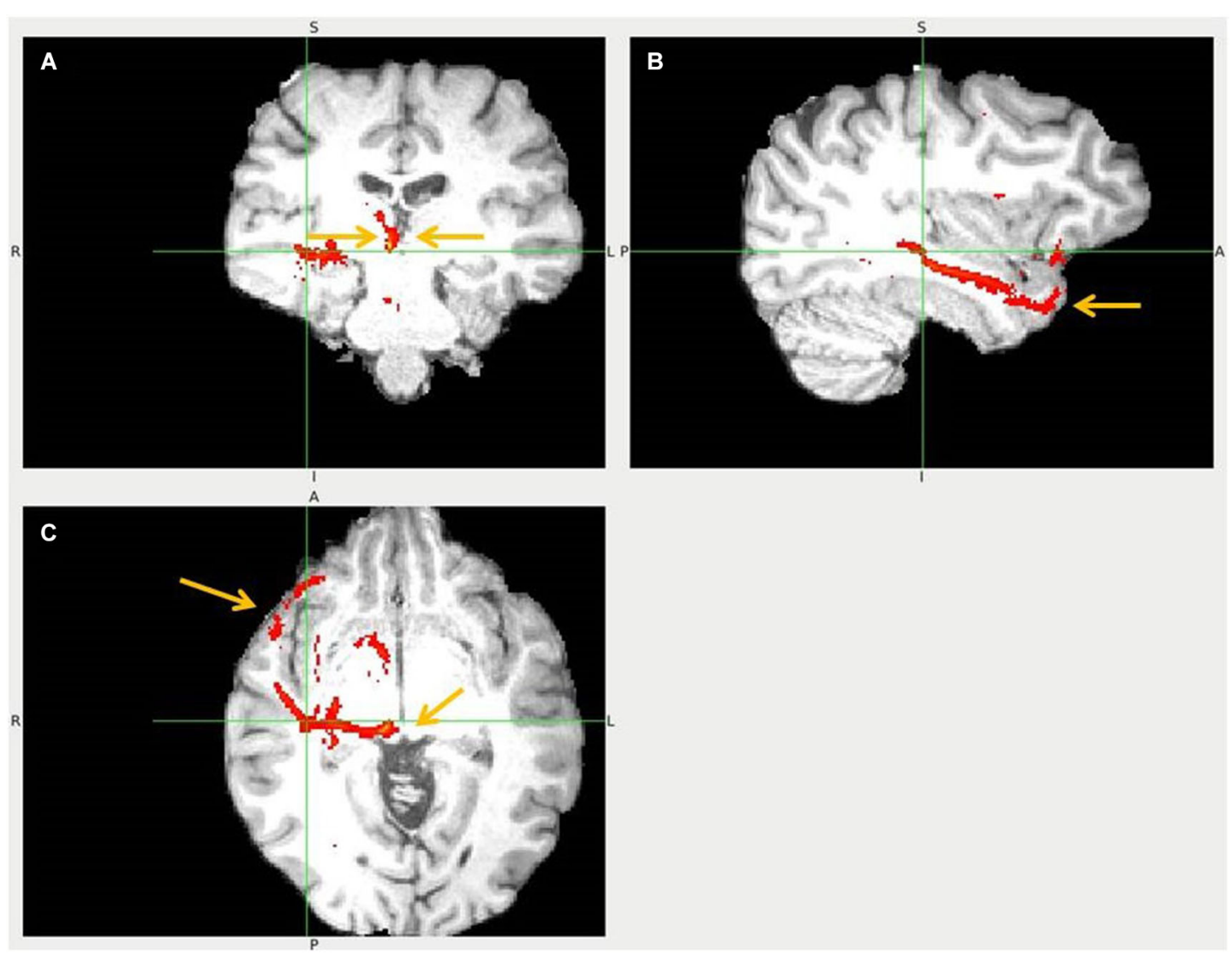

FIGURE 15 | Temporal pole to LHb fiber tract. (A) The arrows point to habenulae. its tract towards superior colliculus and up to LHb.

rules or algorithms are learned by the D1 loop of caudate head potentiation by increased dopamine, after the evidence proved them right/correct. The evidence for wrongness of the same prediction, hypothesis or the way of solving task is inferred from the strength of the glutamatergic synapses on the D2 loop of caudate head, which summate the experienced negative outcomes or failures, errors (for example in grammar rules learning in humans or in instrumental learning in animals). So the evidence "for" vs. "against" the validity/correctness of the current prediction, guess, strategy, model or rule is memorized by the D1 vs. D2 loop strength, to learn the probabilities of being right or wrong or doing things the right or wrong way (in given context, situation).

In addition, the affective and cognitive prefrontal input to $\mathrm{LHb}$ possibly causes de-selection of the non-valuable choices and information from the working memory by suppressing dopamine release in the PFC. So the LHb activation caused by $\mathrm{PFC}$ input might decrease the dopamine signal (from $\mathrm{SNc}$ ) in the PFC towards boring or irrelevant, non-significant information, deselecting the representation of uninteresting information in working memory, to occupy our attention with more useful information - depending on subjective values, motivations, priorities, intentions and goals. The useful, relevant, meaningful information induce dopamine release from $\mathrm{SNc}$, to direct us toward interesting and informationally valuable stuff (i.e., information of predictive value). In accordance with this, the SNc projects to the cognitive PFC and dorsal striatum (Porrino and Goldman-Rakic, 1982; Haber et al., 2000) and the TMS stimulation of dorsolateral PFC (Strafella et al., 2001) induces dopamine release in the caudate nucleus.

Finding direct cognitive PFC projections to $\mathrm{LHb}$ means there might be some prefrontal neuronal populations that preferentially project to the $\mathrm{LHb}$ and other that project to $\mathrm{SNc}$, depending on the value, meaning, significance or usefulness of the processed information for current goal or task. By their $\mathrm{SNc}$ and $\mathrm{LHb}$ efferents, the cognitive PFC regions bias our learning, selection and de-selection of information depending on their meaning and predictive value for current goal/aim, task or context.

\section{CONCLUSIONS}

Using DTI probabilistic tractography I confirmed the cortical input to LHb in humans from the affective regions: AI, clOFC, IOFC, dACC and pgACC, linked to inhibitory self-control and avoidance learning. As predicted I found no LHb projection from vACC. Unexpected findings were the robust projections to the $\mathrm{LHb}$ from the TP and from the cognitive prefrontal regions: BA 10 medial and lateral, BA 9, 8, 44, 45, 46 and 47. 


\section{REFERENCES}

Abulafia, R., Zalkind, V., and Devor, M. (2009). Cerebral activity during the anesthesia-like state induced by mesopontine microinjection of pentobarbital. J. Neurosci. 29, 7053-7064. doi: 10.1523/JNEUROSCI.1357-08.2009

Agetsuma, M., Aizawa, H., Aoki, T., Nakayama, R., Takahoko, M., Goto, M., et al. (2010). The habenula is crucial for experience-dependent modification of fear responses in zebrafish. Nat. Neurosci. 13, 1354-1356. doi: 10.1038/nn.2654

Aggleton, J. P., Vann, S. D., and Saunders, R. C. (2005). Projections from the hippocampal region to the mamillary bodies in macaque monkeys. Eur. J. Neurosci. 22, 2519-2530. doi: 10.1111/j.1460-9568.2005.04450.x

Alexander, G. E., DeLong, M. R., and Strick, P. L. (1986). Parallel organization of functionally segregated circuits linking basal ganglia and cortex. Annu. Rev. Neurosci. 9, 357-381. doi: 10.1146/annurev.neuro.9.1.357

Amat, J., Matus-Amat, P., Watkins, L. R., and Maier, S. F. (1998). Escapable and inescapable stress differentially alter extracellular levels of 5-HT in the basolateral amygdala of the rat. Brain Res. 812, 113-120. doi: 10.1016/s00068993(98)00960-3

Asaad, W. F., Rainer, G., and Miller, E. K. (2000). Task specific neural activity in the primate prefrontal cortex. J. Neurophysiol. 84, 451-459.

Behrens, T. E. J., Berg, H. J., Jbabdi, S., Rushworth, M. F., and Woolrich, M. W. (2007). Probabilistic diffusion tractography with multiple fiber orientations: what can we gain? Neuroimage 34, 144-155. doi: 10.1016/j.neuroimage.2006. 09.018

Behrens, T. E. J., Woolrich, M. W., Jenkinson, M., Johansen-Berg, H., Nunes, R. G., Clare, S., et al. (2003). Characterization and propagation of uncertainty in diffusion-weighted MR imaging. Magn. Reson. Med. 50, 1077-1088. doi: 10. 1002/mrm.10609

Brody, A. L., Saxena, S., and Stoessel, P. (2001). Regional brain metabolic changes in patients with major depression treated with either paroxetine or interpersonal therapy: preliminary findings. Arch. Gen. Psychiatry 58, 631-640. doi: 10. 1001/archpsyc.58.7.631

Chiba, T., Kayahara, T., and Nakano, K. (2001). Efferent projections of infralimbic and prelimbic areas of the medial prefrontal cortex in the Japanese monkey, Macaca fuscata. Brain Res. 888, 83-101. doi: 10.1016/s0006-8993(00) 03013-4

Christoph, G., Leonzio, R., and Wilcox, K. (1986). Stimulation of the lateral habenula inhibits dopamine-containing neurons in the substantia nigra and ventral tegmental area of the rat. J. Neurosci. 6, 613-619.

Drevets, W. C., Spitznagel, E. L., MacLeod, A. K., and Raichle, M. E. (1992). "Discriminatory capability of PET measurements of regional blood flow in familial pure depressive disease," Abstract Presented at Society of Neuroscience Annual Meeting (Anaheim, CA), 18, 1596.

Evans, A. C., Collins, D. L., Mills, S. R., Brown, E. D., Kelly, R. L., and Peters, T. M. (1993). "3D statistical neuroanatomical models from 305 MRI volumes," in Proc. IEEE-Nuclear Science Symposium and Medical Imaging Conference (San Francisco), 1813-1817.

Frank, M., and Hutchison, K. (2009). Genetic contributions to avoidance-based decisions: striatal D2 receptor polymorphisms. Neuroscience 164, 131-140. doi: 10.1016/j.neuroscience.2009.04.048

George, M. S., Ketter, T. A., Parekh, P. I., Horwitz, B., Herscovitch, P., and Post, R. M. (1995). Brain activity during transient sadness and happiness in healthy women. Am. J. Psychiatry 152, 341-351.

Gottesfeld, Z. (1983). Origin and distribution of noradrenergic innervation in the habenula: a neurochemical study. Brain Res. 275, 299-304. doi: 10.1016/00068993(83)90990-3

Grabenhorst, F., and Rolls, E. T. (2011). Value, pleasure and choice in the ventral prefrontal cortex. Trends Cogn. Sci. 15, 56-67. doi: 10.1016/j.tics.2010.12.004

Greatrex, R. M., and Phillipson, O. T. (1982). Demonstration of synaptic input from prefrontal cortex to the habenula in the rat. Brain Res. 238, 192-197. doi: 10. 1016/0006-8993(82)90782-x

Groenewegen, H. J., Ahlenius, S., Haber, S. N., Kowall, N. W., and Nauta, W. J. (1986). Cytoarchitecture, fiber connections and some histochemical aspects of the interpeduncular nucleus in the rat. J. Comp. Neurol. 249, 65-102. doi: 10. 1002/cne.902490107

Guilding, C., and Piggins, H. D. (2007). Challenging the omnipotence of the suprachiasmatic timekeeper: are circadian oscillators present throughout the mammalian brain? Eur. J. Neurosci. 25, 3195-3216. doi: 10.1111/j.1460-9568. 2007.05581.x
Haber, S. N., Fudge, J. L., and McFarland, N. R. (2000). Striatonigrostriatal pathways in primates form an ascending spiral from the shell to the dorsolateral striatum. J. Neurosci. 20, 2369-2382.

Hamani, C., Diwan, M., Macedo, C. E., Brandão, M. L., Shumake, J., GonzalezLima, F., et al. (2010). Antidepressant-like effects of medial prefrontal cortex deep brain stimulation in rats. Biol. Psychiatry 67, 117-124. doi: 10.1016/j. biopsych.2009.08.025

Herkenham, M. (1981). Anesthetics and the habenulo-interpeduncular system: selective sparing of metabolic activity. Brain Res. 210, 461-466. doi: 10. 1016/0006-8993(81)90927-6

Herkenham, M., and Nauta, W. J. (1977). Afferent connections of the habenular nuclei in the rat. A horseradish peroxidase study, with a note on the fiber-ofpassage problem. J. Comp. Neurol. 173, 123-145. doi: 10.1002/cne.901730107

Herkenham, M., and Nauta, W. J. (1979). Efferent connections of the habenular nuclei in the rat. J. Comp. Neurol. 187, 19-47. doi: 10.1002/cne.901870103

Hikosaka, O. (2010). The habenula: from stress evasion to value-based decisionmaking. Nat. Rev. Neurosci. 11, 503-513. doi: 10.1038/nrn2866

Hobson, J. A., McCarley, R. W., and Wyzinski, P. W. (1975). Sleep cycle oscillation: reciprocal discharge by two brainstem neuronal groups. Science 189, 55-58. doi: $10.1126 /$ science. 1094539

Hong, S., and Hikosaka, O. (2008). The globus pallidus sends reward-related signals to the lateral habenula. Neuron 60, 720-729. doi: 10.1016/j.neuron.2008. 09.035

Hong, S., Jhou, T. C., Smith, M., Saleem, K. S., and Hikosaka, O. (2011). Negative reward signals from the lateral habenula to dopamine neurons are mediated by rostromedial tegmental nucleus in primates. J. Neurosci. 31, 11457-11471. doi: 10.1523/JNEUROSCI.1384-11.2011

Jacobs, B. L., and Azmitia, E. C. (1992). Structure and function of the brain serotonin system. Physiol. Rev. 2, 165-229.

Jenkinson, M., Bannister, P. R., Brady, J. M., and Smith, S. M. (2002). Improved optimisation for the robust and accurate linear registration and motion correction of brain images. Neuroimage 17, 825-841. doi: 10.1006/nimg.2002.1132

Jenkinson, M., Beckmann, C. F., Behrens, T. E., Woolrich, M. W., and Smith, S. M. (2012). FSL. Neuroimage 62, 782-790. doi: 10.1016/j.neuroimage.2011.09.015

Jenkinson, M., and Smith, S. M. (2001). A global optimisation method for robust affine registration of brain images. Med. Image Anal. 5, 143-156. doi: 10. 1016/s1361-8415(01)00036-6

Jhou, T. C., Geisler, S., Marinelli, M., Degarmo, B. A., and Zahm, D. S. (2009). The mesopontine rostromedial tegmental nucleus: a structure targeted by the lateral habenula that projects to the ventral tegmental area of Tsai and substantia nigra compacta. J. Comp. Neurol. 513, 566-596. doi: 10.1002/cne.21891

Kim, U., and Chang, S. (2005). Dendritic morphology, local circuitry and intrinsic electrophysiology of neurons in the rat medial and lateral habenular nuclei of the epithalamus. J. Comp. Neurol. 483, 236-250. doi: 10.1002/cne.20410

Kim, U., and Lee, T. (2012). Topography of descending projections from anterior insular and medial prefrontal regions to the lateral habenula of the epithalamus in the rat. Eur. J. Neurosci. 35, 1253-1269. doi: 10.1111/j.1460-9568.2012. 08030.x

Kiss, J., Csáki, A., Bokor, H., Kocsis, K., and Kocsis, B. (2002). Possible glutamatergic/aspartatergic projections to the supramammillary nucleus and their origins in the rat studied by selective $[(3) \mathrm{H}] \mathrm{D}$-aspartate labelling and immunocytochemistry. Neuroscience 111, 671-691. doi: 10.1016/s0306-4522(02) 00037-4

Li, B., Piriz, J., Mirrione, M., Chung, C., Proulx, C. D., Schulz, D., et al. (2011). Synaptic potentiation onto habenula neurons in the learned helplessness model of depression. Nature 470, 535-539. doi: 10.1038/nature09742

Marrosu, F., Fornal, C. A., Metzler, C. W., and Jacobs, B. L. (1996). 5-HT 1A agonists induce hippocampal theta activity in freely moving cats: role of presynaptic 5-HT 1A receptors. Brain Res. 739, 192-200. doi: 10.1016/s0006-8993(96) 00826-8

Matsumoto, M., and Hikosaka, O. (2009). Representation of negative motivational value in the primate lateral habenula. Nat. Neurosci. 12, 77-84. doi: 10.1038/nn. 2233

McCarley, R. W., and Hobson, J. A. (1975). Neuronal excitability modulation over the sleep cycle: a structural and mathematical model. Science 189, 58-60. doi: 10. $1126 /$ science. 1135627

McCormick, D. A., and Prince, D. A. (1987). Actions of acetylcholine in the guineapig and cat medial and lateral geniculate nuclei, in vitro. J. Physiol. 392, 147-165. 
Mirrione, M. M., Schulz, D., Kyle, A., Lapidus, B., Zhang, S., Goodman, W., et al. (2014). Increased metabolic activity in the septum and habenula during stress is linked to subsequent expression of learned helplessness behavior. Front. Hum. Neurosci. 8:29. doi: 10.3389/fnhum.2014.00029

O’Doherty, J., Kringelbach, M. L., Rolls, E. T., Hornak, J., and Andrews, C. (2001). Abstract reward and punishment in the human orbitofrontal cortex. Nat. Neurosci. 4, 95-102. doi: 10.1038/82959

Ongür, D., Ferry, A. T., and Price, J. L. (2003). Architectonic subdivision of the human orbital and medial prefrontal cortex. J. Comp. Neurol. 460, 425-449. doi: 10.1002/cne.10609

Onodera, K., Yamatodani, A., Watanabe, T., and Wada, H. (1994). Neuropharmacology of the histaminergic neuron system in the brain and its relationship with behavioral disorders. Prog. Neurobiol. 42, 685-702. doi: 10.1016/03010082(94)90017-5

Pace-Schott, E. F., and Hobson, J. A. (2002). The neurobiology of sleep: genetics, cellular physiology and subcortical networks. Nat. Rev. Neurosci. 3, 591-605. doi: 10.1038/nrn895

Peyron, C., Petit, J. M., Rampon, C., Jouvet, M., and Luppi, P. H. (1998). Forebrain afferents to the rat dorsal raphe nucleus demonstrated by retrograde and anterograde tracing methods. Neuroscience 82, 443-468. doi: 10.1016/s03064522(97)00268-6

Pizzagalli, D. A., Oakes, T. P., Fox, A. S., Chung, M. K., Larson, C. L., Abercrombie, H. C., et al. (2004). Functional but not structural subgenual prefrontal cortex abnormalities in melancholia. Mol. Psychiatry 9, 393-405. doi: 10.1038/sj.mp. 4001469

Porrino, L., and Goldman-Rakic, P. S. (1982). Brainstem innervation of prefrontal and anterior cingulate cortex in the rhesus monkey revealed by retrograde transport of HRP. J. Comp. Neurol. 205, 63-76. doi: 10.1002/cne.902050107

Qin, C., and Luo, M. (2009). Neurochemical phenotypes of the afferent and efferent projections of the mouse medial habenula. Neuroscience 161, 827-837. doi: 10. 1016/j.neuroscience.2009.03.085

Quick, M. W., Ceballo, R. M., Kasten, M., McIntosh, J. M., and Lester, R. A. (1999). $\alpha 3 \beta 4$ subunit-containing nicotinic receptors dominate function in rat medial habenula neurons. Neuropharmacology 38, 769-783. doi: 10.1016/s00283908(99)00024-6

Quirk, G. J., Likhtik, E., Pelletier, J. G., and Paré, D. (2003). Stimulation of medial prefrontal cortex decreases the responsiveness of central amygdala output neurons. J. Neurosci. 23, 8800-8807.

Rainer, G., Asaad, W. F., and Miller, E. K. (1998). Selective representation of relevant information in primate prefrontal cortex. Nature 393, 577-579. doi: 10. $1038 / 31235$

Rønnekleiv, O. K., and Møller, M. (1979). Brain-pineal nervous connections in the rat: an ultrastructure study following habenular lesion. Exp. Brain Res. 37, 551562. doi: 10.1007/bf00236823

Shabel, S. J., Proulx, C. D., Trias, A., Murphy, R. T., and Malinow, R. (2012). Input to the lateral habenula from the basal ganglia is excitatory, aversive, and suppressed by serotonin. Neuron 74, 475-481. doi: 10.1016/j.neuron.2012.02.037

Shelton, L., Pendse, G., Maleki, N., Moulton, E. A., Lebel, A., Becerra, L., et al. (2012). Mapping pain activation and connectivity of the human habenula. $J$. Neurophysiol. 107, 2633-2648. doi: 10.1152/jn.00012.2012

Simon-Thomas, E. R., Role, K. O., and Knight, R. T. (2005). Behavioral and electrophysiological evidence of a right hemisphere bias for the influence of negative emotion on higher cognition. J. Cogn. Neurosci. 17, 518-529. doi: 10. 1162/0898929053279504

Smith, S. M., Jenkinson, M., Woolrich, M. W., Beckmann, C. F., Behrens, T. E. J., Johansen-Berg, H., et al. (2004). Advances in functional and structural MR image analysis and implementation as FSL. Neuroimage 23, S208-S219. doi: 10. 1016/j.neuroimage.2004.07.051

Strafella, A. P., Paus, T., Barrett, J., and Dagher, A. (2001). Repetitive transcranial magnetic stimulation of the human prefrontal cortex induces dopamine release in the caudate nucleus. J. Neurosci. 21, RC157-RC164.

Sugama, S., Cho, B. P., Baker, H., Joh, T. H., Lucero, J., and Conti, B. (2002). Neurons of the superior nucleus of the medial habenula and ependymal cells express IL-18 in rat CNS. Brain Res. 958, 1-9. doi: 10.1016/s0006-8993(02) 03363-2

Ullsperger, M., and von Cramon, D. Y. (2003). Error monitoring using external feedback: specific roles of the habenular complex, the reward system and the cingulate motor area revealed by functional magnetic resonance imaging. $J$. Neurosci. 23, 4308-4314.

Vadovičová, K., and Gasparotti, R. (2013). Reward and adversity processing circuits, their competition and interactions with dopamine and serotonin signaling. ScienceOpen Research arXiv:1304.4201. doi: 10.14293/S2199-1006.1.SORLIFE.AEKZPZ.v1

van Nieuwenhuijzen, P. S., McGregor, I. S., and Hunt, G. E. (2009). The distribution of $\gamma$-hydroxybutyrate-induced Fos expression in rat brain: comparison with baclofen. Neuroscience 158, 441-445. doi: 10.1016/j.neuroscience.2008. 10.011

Vanni-Mercier, G., Sakai, K., and Jouvet, M. (1984). "Waking-state specific" neurons in the caudal hypothalamus of the cat. C R Acad. Sci. III 298, 195-200.

Vertes, R. P. (2004). Differential projections of the infralimbic and prelimbic cortex in the rat. Synapse 51, 32-58. doi: 10.1002/syn.10279

Vertes, R. P., and Kocsis, B. (1997). Brainstem-diencephalo-septohippocampal systems controlling the theta rhythm of the hippocampus. Neuroscience 81 , 893-926.

Wang, R. Y., and Aghajanian, G. K. (1977). Physiological evidence for habenula as major link between forebrain and midbrain raphe. Science 197, 89-91. doi: 10. 1126/science. 194312

Woolrich, M. W., Jbabdi, S., Patenaude, B., Chappell, M., Makni, S., Behrens, T., et al. (2009). Bayesian analysis of neuroimaging data in FSL. Neuroimage 45, S173-S186. doi: 10.1016/j.neuroimage.2008.10.055

Yu, E. Z., Hallenbeck, J. M., Cai, D., and McCarron, R. M. (2002). Elevated arylalkylamine-N.-acetyltransferase (AA-NAT) gene expression in medial habenular and suprachiasmatic nuclei of hibernating ground squirrels. Brain Res. Mol. Brain Res. 102, 9-17. doi: 10.1016/s0169-328x(02) 00138-9

Conflict of Interest Statement: The author declares that the research was conducted in the absence of any commercial or financial relationships that could be construed as a potential conflict of interest.

Received: 18 February 2014; accepted: 25 September 2014; published online: 27 October 2014.

Citation: Vadovičová $K$ (2014) Affective and cognitive prefrontal cortex projections to the lateral habenula in humans. Front. Hum. Neurosci. 8:819. doi: 10.3389/fnhum.2014.00819

This article was submitted to the journal Frontiers in Human Neuroscience.

Copyright (c) 2014 Vadovičová. This is an open-access article distributed under the terms of the Creative Commons Attribution License (CC BY). The use, distribution and reproduction in other forums is permitted, provided the original author(s) or licensor are credited and that the original publication in this journal is cited, in accordance with accepted academic practice. No use, distribution or reproduction is permitted which does not comply with these terms. 